J3eA, Journal sur l'enseignement des sciences et technologies de l'information et des systèmes, Volume 4, Hors-Série 2, 29 (2005)

DOI : http://dx.doi.org/10.1051/bib-j3ea:2005729

(C) EDP Sciences, 2005

Pédagogie par projet et transfert de technologie : l'exemple du Centre de Recherche, d'Étude et de Développement (CRED) de l'IUT Bordeaux 1

G. Gramaccia, A. Perret et C. Pellet

Centre de Recherche, d'Études et Développement de l'IUT Bordeaux 1

Domaine universitaire

F-33405 Talence, France 


\title{
Pédagogie par projet et transfert de technologie : l'exemple du Centre de Recherche, d'Etude et de Développement (CRED) de l'IUT Bordeaux 1
}

\author{
Gino Gramaccia, Amélie Perret, Claude Pellet, Centre de Recherche, d'Etudes et de \\ Développement de l'IUT Bordeaux 1, Domaine Universitaires 33405 Talence \\ gino.gramaccia@iut.u-bordeaux1.fr, amelie.perret@iut.u-bordeaux1.fr, \\ claude.pellet@iut.u-bordeaux1.fr
}

\begin{abstract}
Résumé :
Cet article fait la synthèse théorique d'une pratique pédagogique engagée depuis une douzaine d'années au sein de l'IUT Bordeaux 1, au Centre de Recherche, d'Etudes et de Développement (CRED), dans le contexte particulier d'une Licence Professionnelle en Gestion de Projets Innovants (LPGPI). Cette structure a fait d'emblée le choix d'associer le réseau des laboratoires universitaires de recherche bordelais et le tissu des entreprises régionales, principalement des PME, pour des opérations de transfert de connaissances scientifiques et technologiques. L'étudiant, titulaire d'un bac+2, est impliqué dans un projet de recherche technologique qu'il doit conduire en autonomie tuteurée, conjointement encadré par un chercheur universitaire et un ingénieur d'entreprise. Ce cadre pédagogique, en constante évolution depuis l'origine, s'appuie sur quelques grands principes dont nous résumons ci-dessous l'essentiel.
\end{abstract}

Mots clés : pédagogie par projet, management de projet, interdisciplinarité, apprentissage.

\section{LE DISPOSITIF DE FORMATION}

Les relations science/industrie ont fait l'objet de multiples approches dans le champ des sciences sociales (sociologie, gestion, économie...). L'hypothèse de base commune à l'ensemble de ces sciences considère la science dite «dure » comme une institution non isolée de la demande sociale, une science non repliée dans le secret des laboratoires. Dans cette logique, la valeur des savoirs produits est estimée d'après leur contribution à la réalisation d'objectifs industriels $[1,2] . \quad \mathrm{La}$ recherche technologique, dont la notion est actuellement en débat, est sans doute l'expression la plus empirique et la plus instable de cette évolution. L'un des traits significatifs de cette recherche est son inscription dans des réseaux de diffusion de connaissances [3]. Ces réseaux associent de multiples partenaires : des chercheurs universitaires, des ingénieurs et des techniciens des universités et de l'industrie et surtout des étudiants dans le cadre de projets de formation à double finalité :

- Une finalité d'apprentissage aux méthodes de la recherche technologique s'inspirant des méthodes et des outils du management de projet.

- Une finalité industrielle qui s'inscrit d'emblée dans la stratégie des entreprises partenaires.

Une telle orientation, mi-pédagogique miindustrielle, suppose un changement de contexte, un parti-pris d'ouverture aux contingences, aux événements, aux aléas. Le risque devient un matériau éducatif. Ce sont les conditions les plus réalistes pour l'exercice de la responsabilité et de l'autonomie. Le passage d'un contexte clos, régulé et contrôlé par les normes et les standards de l'institution à un contexte ouvert à l'incertitude a pour vocation, au moyen d'une pédagogie adaptée, d'inciter l'étudiant à la pratique du diagnostic et aux logiques d'anticipation [4].

Le management de projet fournit la réponse méthodologique la plus appropriée pour la maîtrise de processus complexes qui s'inscrivent à la fois dans le champ de l'innovation technologique, de l'économie et de l'enseignement. Les méthodes et les outils du management de projet ont pris une place légitime dans une pédagogie du/par projet. Il s'agit de maîtriser tous les processus constitutifs d'un projet : analyse des coûts et des temps, étude de faisabilité, analyse des risques, structuration des rôles et des activités et mise en scène de différents rituels de communication (rencontres en face à face, exposés, démonstrations...) pour mieux symboliser les phases d'avancement et d'achèvement du projet. Cette discipline a trouvé son plein emploi méthodologique dans un système complexe et global d'incitations à la professionnalisation des connaissances [5].

Cette approche est à l'œuvre dans les projets de R.T. et la pédagogie associée du Centre de Recherche, d'Etudes et de Développement (le C.R.E.D.) implanté à l'IUT Bordeaux 1 depuis 1991 [6, 7]. Le C.R.E.D. est un centre pluridisciplinaire post-DUT qui a pour mission de former des chefs de projets en R\&D pour les PME/PMI. Il a pour mission d'assurer des actions de transferts de technologies, via un programme de formation à la gestion de projet à bac+3 (licence professionnelle), entre les laboratoires de recherche universitaire et le tissu des PME de technologie avancée de la région Aquitaine. Il forme un cadre organisationnel suffisamment souple pour accueillir des projets d'innovation 
regroupant des chercheurs et des industriels curieux d'évaluer la faisabilité d'un produit nouveau. Les activités projet du C.R.E.D. interviennent dans des structures spécifiques associant des chercheurs, des développeurs, des partenaires industriels (des PME/PMI), une équipe d'étudiants coordonnée par un élève chef de projet. Un stage de 16 semaines au sein de l'entreprise d'accueil est dédié à la conception et à la réalisation d'un prototype (voir les figures 1 et 2). En plus de dix ans, près de trois cents petits projets d'innovation de toute nature technologique ont été ainsi réalisés [8, 9, 10, 11]. Un tel dispositif prend globalement la forme de multiples collaborations pédagogiques avec l'entreprise : double encadrement des stages (par des enseignants et des professionnels), formations continues, enseignements assurés par des ingénieurs).

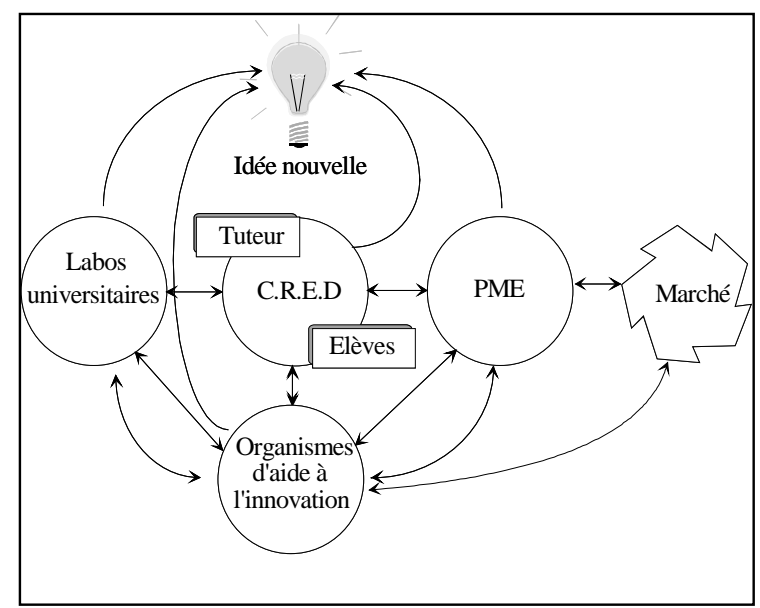

fig 1 : Réseau des composantes dans un projet CRED

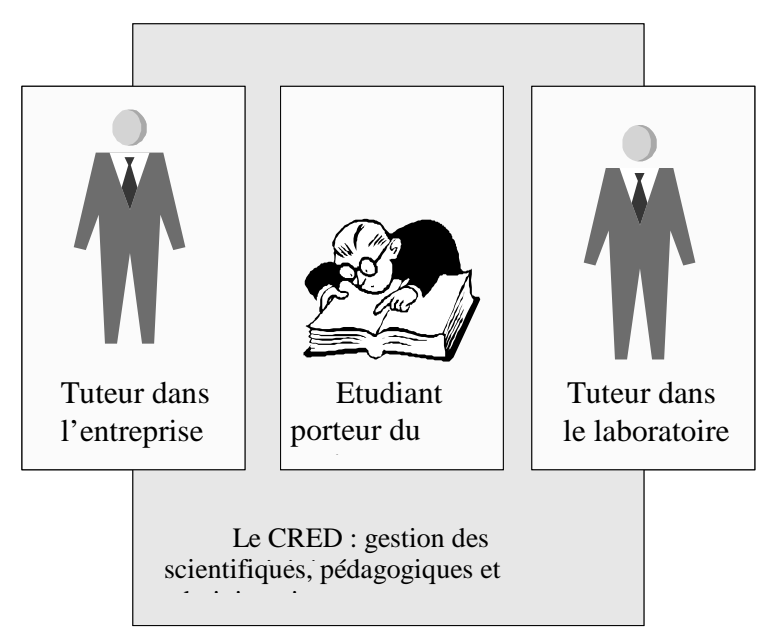

fig 2 : Réseau des composantes dans un projet CRED

\section{LES PRINCIPES A L'GUVRE DANS NOTRE PEDAGOGIE}

Le premier principe est d'avoir considéré diverses notions réputées négatives (le risque, l'incertitude, les contraintes de gestion) comme des éléments positifs de l'action. Ce point de vue n'est pas nouveau dans les IUT mais nous nous sommes efforcés d'en développer les aspects réalistes compte tenu des opportunités de notre contexte industriel et universitaire. Les conditions du risque sont certaines dans notre démarche puisqu'il convient de maîtriser (donc de rendre homogène et efficace) un processus d'innovation dans des contextes interférents et hétérogènes, limitant de fait le calcul des choix rationnels. La difficulté majeure dans ce type d'approche aura été d'intégrer dans un même modèle générique des paramètres hétérogènes qu'on peut résumer en trois catégories : des connaissances fondamentales issues des laboratoires de recherche universitaire, des contraintes de gestion imposées par les nécessités stratégiques de l'entreprise partenaire (objectifs techniques, coûts, délais, qualité) et des objectifs d'apprentissage à la recherche-action. Les principes auxquels nous nous sommes conformés sont détaillés ci-dessous (voir figure 3).

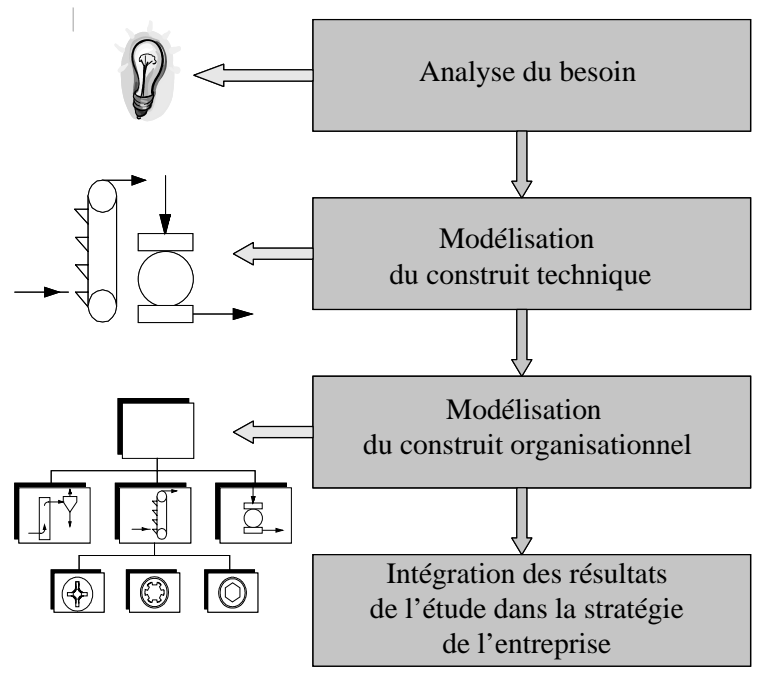

fig 3 : Les niveaux de rationalité dans le processus de transfert de connaissances

\subsection{La recherche d'une justification économique}

La construction des réseaux de coopération associant des acteurs aux attentes diversifiées est contrainte par la relation client/fournisseur. L'analyse du besoin constitue le premier niveau de rationalité. Elle détermine les objectifs économiques du projet et permet d'identifier les parties-prenantes du contrat associées le temps d'une action spécifique jusqu'aux résultats concrets de l'étude. L'étudiant, considéré comme «porteur du projet», est au centre d'un dispositif qui se justifie par une proximité et une solidarité de fait entre l'institution et les entreprises 
régionales (en particulier des PME), encouragées par divers organismes institutionnels d'aide à l'innovation (l'ANVAR, par exemple). Cette proximité est grande puisque de nombreuses PME recrutent en priorité les jeunes diplômés. La solidarité est ici l'expression première de l'éthique qui veut que les connaissances technologiques de pointe soient au plus tôt mises au service des dirigeants de PME (48\% des emplois des titulaires de la LP GPI sont fournis par le secteur industriel).

\subsection{La construction d'une rationalité fonctionnelle}

Nous désignerons sous le terme général de construit technique (un schéma de carte électronique et le descriptif technique associé, par exemple) les concepts descriptifs d'un modèle opératoire chargé de rendre compte du fonctionnement reproductible d'un système, d'un procédé ou d'un processus. Ce construit forme, dans le processus d'ensemble du transfert, un premier niveau de rationalité. Il est caractérisé par son autonomie de fonctionnement et peut faire l'objet d'une modélisation susceptible de correction et de validation. Dans sa forme aboutie, il correspond au prototype.

\subsection{La construction d'une cohérence organisationnelle}

Ce qui est transféré, c'est moins le construit premier (le prototype) que ses déclinaisons successives à différents paliers de rationalité (organisationnel, commercial, communicationnel, financier...) dont l'intégration constitue au final le vecteur de diffusion des connaissances. Un savoir procédural est alors nécessaire pour contrôler et modéliser une telle progression : il s'agit, au moyen de différents outils graphiques ou d'objets de simulation (des plannings, des matrices d'organisation, des courbes, des maquettes) de simplifier, de transposer et d'intégrer certains éléments du modèle de construit technique dans un modèle opératoire hétérogène. Un microprocesseur peut être, au moyen d'une schématique appropriée, cet élément/composant susceptible de constituer un élément d'une structure d'organisation. Sa représentation schématique intervient dans la décomposition des tâches pour une équipe de développeurs, par exemple. La méthodologie dont nous nous inspirons est conforme aux préconisations normalisées de l'AFITEP $^{1}$ pour le management de projet. Son apport est très significatif pour la pédagogie centrée sur l'innovation [12].

\subsection{Individualisation des tâches et management d'équipe}

Ces deux approches, apparemment contradictoires, forment deux logiques complémentaires. Ce sont les

\footnotetext{
1 Association Française des Estimateurs, Techniciens et Ingénieurs de Projet.
}

conditions d'apprentissage de l'individualisation qui nous intéressent ici. En premier lieu, l'étudiant est considéré comme un individu concret, avec sa personnalité propre, ses connaissances identifiées et évolutives dans des contextes spécifiques. L'individualisation des tâches correspond alors à la construction de l'autonomie individuelle dans des contextes nécessairement hétérogènes (globalement, l'espace de la recherche universitaire et l'espace industriel) [13]. Le projet et ses objectifs de développement forment le contexte immédiat de cette autonomie et son cortège classique de qualités qui en résultent: initiative, esprit de décision, hypothèses explicatives face à des problématiques complexes...). En second lieu, l'équipe intervient comme un espace organisé au sein duquel, dans l'entreprise ou le laboratoire, l'individu met à l'épreuve de telles qualités [14, 15, 16, 17]. Nous avons introduit, dans notre dispositif de formation, les groupes d'analyse de projet (G.A.P) définis comme des lieux d'échanges et d'analyse ponctuels (trois heures par mois). Les G.A.P sont formés de 7 ou 8 étudiants d'horizons technologiques différents sous la direction d'un enseignant animateur. Il se réunissent chaque mois à l'occasion des sessions de formation théorique. Ils répondent à trois objectifs :

- Faire le point de l'avancement des projets en évaluant ce qui reste à faire compte tenu des événements survenus au cours du mois.

- Fournir un support d'application des connaissances théoriques acquises en cours.

- Former un espace de résolution de problèmes et de propositions de solutions.

De tels groupes remettent au goût du jour les anciens cercles de qualité, initialement conçus comme des outils d'évaluation d'événements et de construction d'une démarche collective et solidaire. Mais leur intérêt principal est de convoquer des savoirs multiples pour l'analyse globale des problèmes rencontrés. Chacun peut, dans le groupe, faire part de ses difficultés dans les termes de sa spécialité (un problème ardu de conception, par exemple). Les G.A.P. rassemblent des projets multidisciplinaires (informatique, sciences des matériaux, biologie, gestion de la qualité...). La nécessité d'une approche interdisciplinaire s'impose donc pour communiquer sur les points critiques du projet.

\subsection{Objectivation du risque et de la complexité}

La confrontation objective aux attributs du risque et de la complexité dans les projets (décisions irréversibles, incertitude forte, influence des variables exogènes... : voir tableau 1) constitue le cinquième principe pédagogique. On sait que la prévention $\mathrm{du}$ risque et la modélisation des processus complexes sont deux préoccupations fortes du management de projet contemporain, notamment dans les projets dits «majeurs» impliquant, pour l'entreprise, une stratégie de rupture par rapport aux activités maîtrisées. Pour 
répondre à des besoins vérifiés mais non exprimés du marché, de tels projets conjuguent plusieurs processus novateurs : développement d'un concept de produit, mise en place d'outils de production adaptés, conception d'une organisation ad hoc, recherche d'un circuit de distribution, engagement de nouvelles ressources... Ces enjeux ouvrent, pour l'étudiant porteur du projet, des perspectives de formation originales mais aussi, nous l'admettons volontiers, paradoxales. L'obligation (relative) de résultat dans une situation industrielle critique l'éloigne du parcours initiatique classique évalué selon le savoir acquis. Directement impliqué dans la gestion d'un risque, autonome dans la recherche d'une solution (traitement de la complexité), il est contraint, à juste titre, de faire valoir sa réalisation de projet à la fois selon des critères universitaires et des critères industriels. Cette «mixité » est toutefois compensée par la production d'un mémoire et d'une soutenance.

\begin{tabular}{|c|c|}
\hline L’activité Projet & L'activité de production \\
\hline $\begin{array}{l}\text { Non répétitive (one shot) } \\
\text { Il s'agit d'activités intervenant dans la réalisation } \\
\text { d'un projet spécifique (un pont, un prototype...). }\end{array}$ & $\begin{array}{l}\text { Répétitive : } \\
\text { Un ensemble d'opérations administratives à un } \\
\text { guichet, des activités de montage sur une chaîne de } \\
\text { production... }\end{array}$ \\
\hline $\begin{array}{l}\text { Décisions irréversibles } \\
\text { Les décisions sont «engageantes »; elles sont autant } \\
\text { plus irréversibles qu'on avance dans le cours du } \\
\text { projet. Tout retour en arrière peut être extrêmement } \\
\text { pénalisant. }\end{array}$ & $\begin{array}{l}\text { Réversibles } \\
\text { Une opération peut progresser selon le processus } \\
\text { essai-erreur-correction-bonne décision. }\end{array}$ \\
\hline $\begin{array}{l}\text { Incertitude forte } \\
\text { L'incertitude est liée à la difficulté de maîtriser les } \\
\text { risques financiers, technologiques, les risques liés à } \\
\text { l'indisponibilité des ressources... }\end{array}$ & $\begin{array}{l}\text { Incertitude faible } \\
\text { Les activités professionnelles routinières sont } \\
\text { globalement prévisibles. }\end{array}$ \\
\hline $\begin{array}{l}\text { Influence forte des variables exogènes } \\
\text { Ces variables ont généralement un effet négatif sur le } \\
\text { déroulement du projet : des catastrophes, l'apparition } \\
\text { d'un concurrent, l'évolution du besoin du client... }\end{array}$ & $\begin{array}{l}\text { Influence forte des variables endogènes } \\
\text { Des pannes sur des processus automatisés, des conflits } \\
\text { collectifs... }\end{array}$ \\
\hline $\begin{array}{l}\text { Processus historiques } \\
\text { Le projet est construit comme un récit, enchaînant des } \\
\text { séquences d'événements. }\end{array}$ & $\begin{array}{l}\text { Processus stabilisés. } \\
\text { Processus gérables au moyen de statistiques } \\
\text { (processus de production, activités métier maîtrisées). }\end{array}$ \\
\hline Cashs flows négatifs & Cashs flows positifs \\
\hline
\end{tabular}

\section{CONCLUSION}

Le management de projet offre assurément de bonnes ressources méthodologiques pour le déploiement industriel de la science et de la technologie. Dans un contexte de formation universitaire, cette discipline fournit les meilleures garanties pour un apprentissage réaliste et réflexif (formation à la recherche par la recherche) des processus de conception et de développement de solutions innovantes. Le CRED a mis en œuvre de telles méthodologies avec le souci constant de corriger et de tirer parti d'erreurs d'ajustement. La formation, systématiquement évaluée par les premiers concernés (les étudiants), mais aussi par les tuteurs et les formateurs, a pu ainsi évoluer sans contraintes au sein de ce qui peut constituer, pour ses acteurs, une plate-forme pédagogique expérimentale, autrement dit un cadre propice à l'innovation pédagogique en même temps qu'un espace de liberté pour la promotion sociale et économique de la science et de la technologie.

\section{Bibliographie}

[1] Gibbons M. et al. (1994). The new production of knowledge. The dynamics of science and research in contemporary societies, London. Voir également Albert M., Bernard P. (2000), « Sous l'empire de la science. Nouvelle production de connaissances et sciences économiques québécoises », in : Sciences de la Société, $\mathrm{n}^{\circ} 49$, p. 27. A une pratique d'évaluation par les pairs (le Mode 1), s'ajoute maintenant, voire se substitue, une logique de Mode 2, qui assigne à la science une finalité pratique.

[2] Grossetti M. (2000). «Sciences et demandes sociales au tournant du siècle », p. 7., Martino 
Nieddu M. (2000). "Science et dynamiques économiques », p. 86, in : Sciences de la Société, $\mathrm{n}^{\circ} 49$.

[3] Callon M. (1986). «Eléments pour une sociologie de la traduction », in: L'Année sociologique, PUF, Paris. Latour B., Woolgar S. (1998), La vie de laboratoire. La production des faits scientifiques, La Découverte, Paris.

[4] Boutinet J.-P (1996). Anthropologie du projet, PUF, p. 209.

[5] Cazaubon C., Gramaccia G., Massard G. (1997). Management de projet technique, Ellipses, Paris.

[6] C. Pellet C., J.F. Bellegarde J.-F., « Le Centre de Recherche Étude et Développement : Structure de formation par la $\mathrm{R} \& \mathrm{D} »$, Colloque sur $\mathrm{La}$ Recherche et le Transfert de Technologie dans les IUT, Saint Nazaire, Février 1995.

[7] http://www-cred.iut.u-bordeaux1.fr

[8] Cazaubon C., Gramaccia G., Pellet C. «Gestion de projet et recherche technologique : une expérience réalisée au CRED de l'IUT Bordeaux 1 », 1er Colloque sur l'Enseignement des Technologies et des Sciences de l'Information et des Systèmes, Orsay, 20-21 novembre 1997.

[9] Cazaubon C., Gramaccia G., Pellet C. "Technology transfers between resarch laboratories and industry: methodological educational choices. Three examples in electronics at the CRED - IUT Bordeaux 1", 9th EAEEIE international conference on Education in Electrical and Information Engineering : Enhancement of education in electrical and information engineering through industry cooperation and research, Lisbonne, 18-20, mai1998.

[10] Gramaccia G., Cazaubon C., Pellet C. « Gérer un projet de transfert de technologie : du besoin au prototype, l'exemple du calcimètre automatique réalisé au C.R.E.D. de l'IUT de l'université Bordeaux 1», 3ème Conférence Internationale sur l'Automatisation Industrielle, Montréal (Canada), juin 1999

[11] http://www-cred.iut.u-bordeaux1.fr/docsArchi/ communication/AnnuairePublicCRED2003.pdf

[12] Chanchevrier M. (MATRA MS21) (1993). «Une définition de l'ingénierie simultanée », in : La Cible n47, pp. 14-16.

[13] Gardère E, G. Gramaccia G., Pellet C., Perret A.. «La communication dans les projets de recherche technologique: l'exemple du CREDIUT Bordeaux 1», Colloque National de la Recherche Universitaire dans les IUT, Tarbes, mai 2003.

[14] Gramaccia G (1999). La communication et les projets, Editions Weka, Paris.

[15] Gramaccia G (2001). Les actes de langage dans les organisations, L'Harmattan, Paris.

[16] Gramaccia G. (2001). Les actes de langage dans les organisations, Paris, L'Harmattan.
[17] Weick K. E. (1995). Sensemaking in Organizations, Sage, Thousands Oaks.

\section{Pour en savoir plus... :}

Giroux H, Taylor J.-R. (1999) «L'évolution du discours sur la qualité : d'une traduction à l'autre », in : Communication \& Organisation $n^{\circ} 15$.

Horvàth P. (1995). «Pour un contrôle de gestion à l'écoute des marchés », in: Revue Française de Gestion $n^{\circ} 104$, pp. 72-85.

Hottois G. (1993), Essais de philosophie bioéthique et biopolitique, Vrin.

Hottois G. (1993), Simondon et la philosophie de la «culture technique», De Boeck Université, Bruxelles.

Lhuillier J.-N. (1993). « Gestion des interfaces », in : La Cible, $n^{\circ} 50$.

Veltz P., Zarifian P.,(1993). «Vers de nouveaux modèles d'organisation », in : Sociologie du travail, XXX 1 . 